Research article

\title{
Intestinal parasitic infections in Thai HIV-infected patients with different immunity status Viroj Wiwanitkit
}

\author{
Address: Department of Laboratory Medicine, Faculty of Medicine, Chulalongkorn University, Bangkok 10330, Thailand \\ E-mail: wviroj@pioneer.netserv.chula.ac.th
}

Published: I June 200I

Received: 19 April 200I

BMC Gastroenterology 200 I, I:3

Accepted: I June 200 I

This article is available from: http://www.biomedcentral.com/I47I-230X/I/3

(c) 200 I Wiwanitkit, licensee BioMed Central Ltd.

\begin{abstract}
Background: One of the major health problems among HIV seropositive patients is superimposed infection due to the defect of immunity. Furthermore, intestinal parasite infection, which is also one of the basic health problems in tropical region, is common in these patients. In this study, a cross sectional study to document the prevalence of intestinal parasitic infection in Thai HIV-infected patients with different immune status was performed.

Methods: A study of stool samples from 60 Thai HIV-infected patients with different immune status was performed at King Chulalongkorn Memorial Hospital, Thailand. Each patient was examined for CD4 count and screened for diarrheal symptoms.

Results: The prevalence of intestinal parasitic infection among the HIV-infected patients in this study was $50 \%$. Non- opportunistic intestinal parasite infections such as hookworms, Opisthorchis viverrini and Ascaris lumbricoides were commonly found in HIV-infected people regardless of immune status with or without diarrheal symptoms. Opportunistic intestinal parasites such as Cryptosporidium, Isospora belli, Microsporidia and Strongyloides stercoralis infection were significantly more frequent in the low immunity group with diarrhea.

Conclusion: Therefore, opportunistic intestinal parasite infection should be suspected in any HIV infected patient with advanced disease presenting with diarrhea. The importance of tropical epidemic non-opportunistic intestinal parasite infections among HIV-infected patients should not be neglected.
\end{abstract}

\section{Background}

Human immunodeficiency virus (HIV) infection, a worldwide infection, is a serious problem in the present day. A high rate of infection is found in many regions of the world, including the Southeast Asia. In Thailand, a tropical country in Southeast Asia, HIV infection is a major problem, like in other regional countries. It has been estimated that a million people are infected with HIV infection in Thailand [1]. And it also the possibly higher undetected HIV infection in the community.
One of the major health problems among HIV seropositive patients is superimposed infection due to the defect of immunity. Furthermore, intestinal parasite infection, which is also one of the basic health problems in tropical region [2], is common in these patients.

In this study, a cross sectional study to document the prevalence of intestinal parasitic infection in Thai HIVinfected patients with different immune status was performed. 
Table I: Prevalence of intestinal parasitic infections in HIV- infected patients.

\begin{tabular}{ccc}
\hline CD 4 T-cell & \multicolumn{2}{c}{ Infected cases/ total cases } \\
\cline { 2 - 3 } categories & With diarrhea & $\begin{array}{c}\text { Without di- } \\
\text { arrhea }\end{array}$ \\
& & \\
\hline $200-499 / \mu \mathrm{L}$ & $3 / 6$ & $2 / 11$ \\
$<200 / \mu \mathrm{L}$ & $5 / 8$ & $3 / 10$ \\
& $12 / 14$ & $5 / 11$ \\
\hline
\end{tabular}

\section{Materials and methods}

This study was performed as a cross-sectional descriptive study. Sixty HIV-infected patients who visited the Out Patient Department, King Chulalongkorn Memorial Hospital during March - April 2000 were included in this study. All revealed no previous medical history of any antiretroviral drug therapy usage. All also did not receive any other protozoa or fungal prophylaxis regimens.

Five milliliters of EDTA blood from each subject was collected for CD4 count. The CD4 count determination in this study was performed by automated flow cytometry analyzer, FACS Calibur (Becton Dickinson). All subjects were categorized by their immune status according to the 1993 revised classification system for HIV infection by CD4 T-cell categories [3].

Stool examination was performed and the patients were screened for a history of diarrhea, which was defined as loose stool occurring more than three times a day. Each collected stool sample was investigated for intestinal parasitic organisms by both simple and concentration technique [4]. Each sample was stained with iodine and modified Trichrome's stain to identify the intestinal parasitic organisms. All data from stool study was collected then analyzed using descriptive statistical analysis.

\section{Results}

During the period of study, 60 stool samples from 60 HIV-infected patients were collected. The study population consisted of 17 patients with $\mathrm{CD} 4>500 / \mu \mathrm{L}, 18$ patients with $\mathrm{CD} 4=200-499 / \mu \mathrm{L}$, and 25 patients with $\mathrm{CD} 4<200 / \mu \mathrm{L}$.

Categorized by CD4 investigation and diarrhea symptom, the prevalence of intestinal protozoa infection is shown in Table 1. Result showed that $50 \%$ of the total subjects were infected with intestinal parasites. No case with polyparasitism was detected in this study. Considering the parasites detected, it revealed that there were many types of organisms as shown in Table 2.

\section{Discussion}

Intestinal parasite infection is an important problem in the HIV-infected patient. With the impaired immunity in these patients, infestation with intestinal parasite organisms resulting in diarrheal symptom is commonly seen $[5,6,7]$. In Thailand, intestinal parasite infection is an important health problem [2]. In this study, the prevalence of intestinal parasite infection among HIV- infected patients with different immune status was investigated. Retrospectively identified from our series, the range $\mathrm{CD} 4$ count and RNA viral load of the subjects were $10-1050 / \mu \mathrm{L}$ and $500-80,000$ copies $/ \mathrm{mL}$, respectively.

Table 2: Intestinal parasites found in stools of HIV- infected patients.

\begin{tabular}{|c|c|c|c|c|c|c|c|}
\hline \multirow{3}{*}{ Organisms } & \multicolumn{7}{|c|}{ Number of infected cases } \\
\hline & \multicolumn{2}{|l|}{$\mathrm{CD} 4 \geq 500 / \mu \mathrm{L}$} & \multicolumn{2}{|l|}{ CD4 $200-499 / \mu \mathrm{L}$} & \multicolumn{2}{|l|}{$\mathrm{CD} 4<200 / \mu \mathrm{L}$} & \multirow[t]{2}{*}{ total } \\
\hline & diarrhea & no & diarrhea & no & diarrhea & no & \\
\hline Hookworm eggs & 3 & 1 & I & 1 & 2 & 0 & 8 \\
\hline Opisthorchis viverrini eggs & 0 & 0 & 2 & 1 & I & 2 & 6 \\
\hline Ascaris lumbricoides eggs & 0 & I & 2 & 0 & 3 & 2 & 8 \\
\hline Cryptosporidium & 0 & 0 & 0 & 0 & 2 & 0 & 2 \\
\hline Isospora belli & 0 & 0 & 0 & 0 & 2 & 1 & 3 \\
\hline Microsporidium parvum & 0 & 0 & 0 & 0 & 1 & 0 & 1 \\
\hline Strongyloides stercolaris larva & 0 & 0 & 0 & $\mathrm{I}$ & 1 & 0 & 2 \\
\hline
\end{tabular}


The prevalence of intestinal parasite among the HIV- infected patients is about $50 \%$. Hookworm and Ascaris lumbricoides appeared to have the highest prevalence ( $13.33 \%$ and $13.33 \%$ ), followed by Opisthorchis viverrini (10 \%), Isospora belli (5\%), Strongyloides stercolaris (3.33\%), Cryptosporidium (3.33\%), and Microsporidium (1.67\%) infection.

Non-opportunistic intestinal parasites such as hookworms, Opisthorchis viverrini and Ascaris lumbricoides were common in HIV-infected patients regardless of immune status. This can state the importance of the common intestinal worm infection in Thailand. While opportunistic intestinal parasites, i.e. Cryptosporidium, Isospora belli, Microsporidium parvum and Strongyloides stercoralis, were common in the low immunity group. Therefore, non-opportunistic intestinal parasitic infection among HIV-infected patients should not be overlook.

The prevalence of intestinal parasites was significantly higher in patients with diarrhea $(2 \mathrm{O} / 28)$ than in those without $(10 / 32)$ (P value < 0.05$)$. Considering the types of organisms, hookworm ( 6 cases) is the most common detected organism in diarrhea group and Opisthorchis viverrini and Ascaris lumbricoides (3 cases and 3 cases) are the two most common detected organisms in non-diarrhea group (Table 1).

Classified by the CD 4 T-cell categories, opportunistic intestinal parasite infections showed a highest prevalence in patients with a low immune level $(\mathrm{CD} 4+<200 / \mu \mathrm{L})$ and diarrhea. There was no significant predominance of non- opportunistic intestinal parasite infections at any immune level. This result shows the importance of the association of opportunistic intestinal infection with diarrheal symptoms in the low immunity group of HIV-infected patients $[5,6,7]$. While non-opportunistic intestinal parasite infections are still an important problem in HIV-infected patients at any immunity level with or without the symptoms of diarrhea.

\section{Conclusion}

It may be concluded that in Thai HIV-infected patients, both opportunistic and non-opportunistic intestinal parasite infections are still highly prevalent. In the management of HIV-infected patients in Thailand with or without diarrhea symptom, stool examination is still a useful investigation. Therefore, as shown in previous reports, opportunistic intestinal parasite infection should be suspected in any HIV-infected patient with low immunity presenting with diarrhea. The importance of tropical epidemic non-opportunistic intestinal parasite infections should not be neglected.

\section{Acknowledgements}

The author has to thank Professor Phairut Deesudchit, Department of Preventive Medicine, Faculty of Medicine, Chulalongkorn University for his advice on writing this article and Associate Professor Suwannee Nithiuthai, Department of Parasitology, Faculty of Veterinarian Science for her advice on each scientific technique.

\section{Competing interests}

None declared

\section{References}

I. Surasiengsunk S, et al: Demographic impact of the HIV epidemic in Thailand. AIDS 1998, 12:775-784

2. Eamsobhana $P$, Boranintra $K$ : Identification of intestinal parasites in the quality assessment programme for the year 1984 - in Thailand. J Med Assoc Thai 1987, 72:I I-15

3. : 1993 revised classification system for HIV infection and expanded surveillance on definition of AIDS among adolescents and adults. Morbid Weekly Rep 1993, 4I: I-19

4. Bray WE: Feces and intestinal parasites. In: Bray, WE. ed. Clinical Laboratory Methods, St Louis: CV Mosby, 1957, 4:368-408

5. Punpoowong B, et al: Opportunistic protozoa in stool samples from HIV-infected patients. Southeast Asian J Trop Med Public Health 1998, 29:31-34

6. Pape JW, et al: Cyclospora infection in adults infected with HIV. Clinical manifestation, treatment and prophylaxis. Ann Intern Med 1994, 12 1:654-657

7. Tarimo DS, et al: Prevalence of intestinal parasites in adult patients with enteropathic. AIDS in North-eastern Tanzania. East African Med J 1996, 73:397-399

\section{Pre-publication history}

The pre-publication history for this paper can be accessed here:

http://www.biomedcentral.com/content/backmatter/ 1471-230x-1-3-b1.pdf

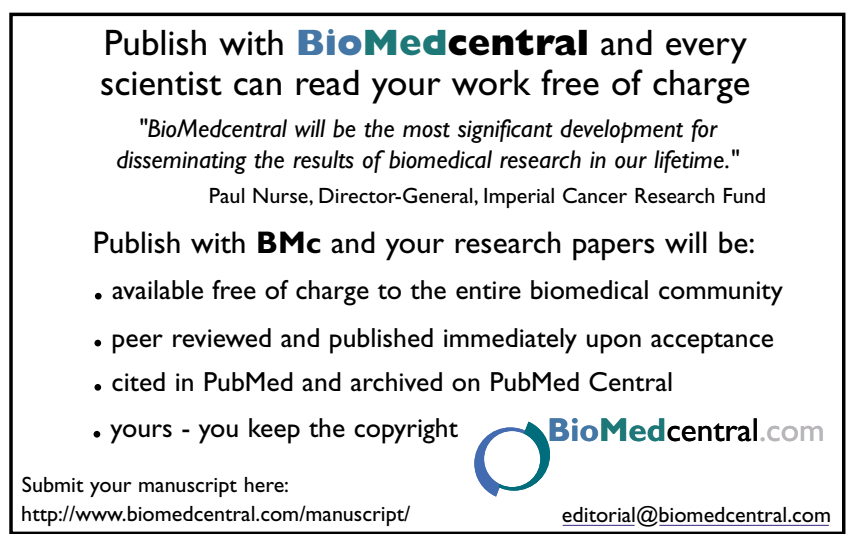

